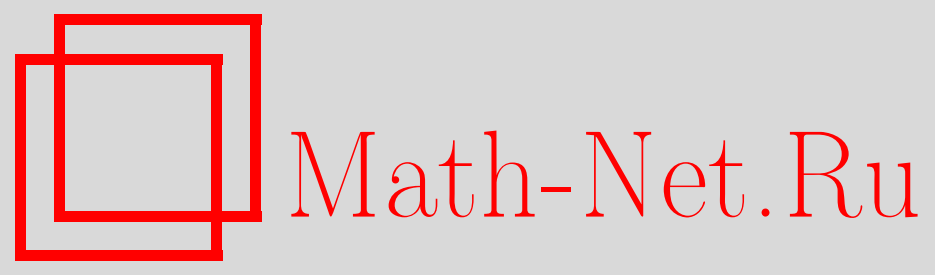

А. А. Ташкинов, В. Е. Шавшуков, Теоретикополевой подход к описанию деформирования многокомпонентных поликристаллических материалов, Вестн. Сам. гос. техн. ун-та. Сер. Физ.мат. науки, 2013, выпуск 4(), 86-97

DOI: https://doi.org/10.14498/vsgtu1282

Использование Общероссийского математического портала MathNet.Ru подразумевает, что вы прочитали и согласны с пользовательским соглашением

http://www.mathnet.ru/rus/agreement

Параметры загрузки:

IP : 54.172 .240 .79

26 апреля 2023 г., 14:09:14

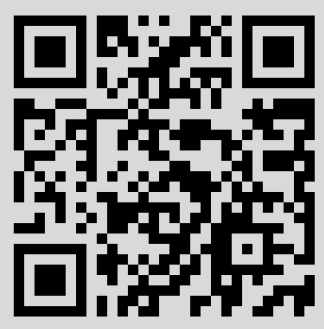


Вестн. Сам. гос. техн. ун-та. Сер. Физ.-мат. науки. 2013. № 4 (33). С. $86-97$

УДК 530.145:539.3/.8

\title{
ТЕОРЕТИКО-ПОЛЕВОЙ ПОДХОД К ОПИСАНИЮ ДЕФОРМИРОВАНИЯ МНОГОКОМПОНЕНТНЫХ ПОЛИКРИСТАЛЛИЧЕСКИХ МАТЕРИАЛОВ
}

\author{
А. А. Ташкинов, В. Е. Шавшуков
}

Пермский национальный исследовательский политехнический университет, Россия, 614990, Пермь, Комсомольский проспект, 29.

E-mails: tash@pstu.ru; shavshukov@pstu.ru

\begin{abstract}
В работе отмечена аналогия между уравнениями механики неоднородной поликристаллической средь и уравнениями квантовой теории рассеяния частии на силовых иентрах, в частности рассеяния электронов проводимости в металлическом сплаве на атомах примесей. Эта аналогия позволяет применить методы решения уравнений квантовой теории поля к решению уравнений механики дебормируемого твёрдого тела для неоднородной среды. Рассмотрено применение метода Корринги-Кона-Ростокера, исполъзуемого в квантовой теории электронов проводимости, для вычисления полей напряжений и дебормаций в многофазном поликристалле. Метод даёт возможность проследить эволюиию напряжённого состояния каждого отдельного кристаллита в процессе макродебормирования, при этом состояние всего поликристаллического тела представляется суперпозищией состояний кристаллитов. В качестве иллюстрации показано, что метод даёт новое решение для классической задачи определения эфбективных модулей упругости неоднородной среды.
\end{abstract}

Ключевые слова: квантовая теория поля, поликристаллы, напряжения, деформации, тензор эффективных модулей упругости.

Введение. Поликристаллические материалы представляют собой систему многих взаимодействующих кристаллитов. Макроскопическое поведение такой системы в конечном счёте определяется свойствами составных элементовкристаллитов, протекающими в них процессами и взаимодействием индивидуальных кристаллитов. Это типичная задача многих тел механики и физики конденсированного состояния, не имеющая точных решений. При рассмотрении деформирования и разрушения поликристаллов на мезоуровне можно считать свойства отдельных кристаллитов известными, описываемыми различными феноменологическими соотношениями (закон Гука, закон Шмида для пластического скольжения, феноменологические критерии разрушения и т.д.) с известными материальными константами.

Многокомпонентные поликристаллические материалы, будучи одними из самых распространённых конструкционных материалов, представляют значительные трудности для описания их поведения при деформировании на мезоуровне методами механики деформируемого твёрдого тела. Причина тому - сложная гетерогенная структура поликристаллов. Даже простейшие краевые задачи линейной теории упругости для таких тел требуют точного задания геометрии всех межфазных границ кристаллитов, отделяющих области с разными упругими модулями, что само по себе является непосильной задачей

Анатолий Александрович Ташкинов (д.ф.-м.н., проф.), ректор; профессор, каф. механики композиционных материалов. Вячеслав Евгенъевич Шавшуков (к.ф.-м.н., доц.), доцент, каф. механики композиционных материалов. 
при макроскопически большом числе кристаллитов. Стохастическая структура этих границ и случайность ориентации кристаллографических осей кристаллитов диктуют применение статистических методов. В современной механике гетерогенных сред для описания стохастических полей деформаций и напряжений традиционным является метод статистических моментов. Согласно этому методу производится осреднение полей по представительному объёму среды. Для поликристаллов использование концепции представительного объёма обычно всегда применимо в силу малых размеров отдельных кристаллитов. Практические возможности метода ограничены вычислением первых статистических моментов случайных полей - обычно средних значений и дисперсий, в редких случаях - моментов более высокого порядка. Но и при вычислении первых моментов требуется принятие различных упрощающих модельных представлений.

Механика деформирования поликристаллических материалов имеет давнюю историю. Первыми работами, в которых была принята во внимание гетерогенная структура поликристаллов и которые не утратили значения для современной науки, можно считать работы Фойгта по упругости поликристаллов [1] и работы Тейлора по пластичности поликристаллов [2]. В них принималось, что деформации во всех зернах поликристалла одинаковы и равны макродеформации представительного объёма, а различие напряжений в разных зернах связано только с различием модулей упругости. Это делало операции осреднения полей напряжений тривиальными. Качественно новым шагом в моделировании поликристаллов явилась работа И. М. Лифшица 1946 года [3]. В ней впервые было учтено различие деформаций в кристаллитах и вычислены эффективные модули упругости поликристалла по известным упругим модулям соответствующих анизотропных монокристаллов во втором порядке по степени анизотропии кристаллитов. Впоследствии это приближение получило название корреляционного приближения. В дальнейшем были разработаны методы осреднения полей напряжений и деформаций с учетом поправок высшего порядка по степени анизотропии - сингулярное приближение, самосогласованное приближение и некоторые другие, обзор которых дан, например, в [4]. Однако все эти работы были посвящены практически одной цели - вычислению эффективных характеристик материала в целом.

Во временных рамках параллельно упомянутым работам в области механики деформируемого твердого тела развивались методы решения задач статистической физики систем многих взаимодействующих частиц - твердых тел, жидкостей, неидеальных газов [5]. В частности, во многих случаях оказалась плодотворной гипотеза представления макроскопической системы сильно взаимодействующих частиц как совокупности слабо взаимодействующих квазичастиц (или элементарных возбуждений), но имеющих перенормированную (эффективную) массу, отличную от массы реальных частиц. Были развиты методы вычисления как макроскопических средних различных физических величин (энергии, теплоемкости и т. д.), так и параметров описания поведения отдельных квазичастиц (энергетического спектра, эффективной массы и др.) и их взаимодействия - относительно слабого в сравнении со взаимодействием реальных атомов и молекул. Значительный прогресс в ста- 
тистической физике был достигнут после установления глубокой аналогии между квантовой теорией поля и статистической физикой. Применение методов, разработанных в теории поля, позволило решить многие задачи физики конденсированного состояния, ранее не разрешимые традиционными методами [6]. Математический аппарат квантовой теории поля имеет несколько различных формулировок. Как будет видно из дальнейшего рассмотрения, к механике гетерогенных материалов математически наиболее близка квантовая теория рассеяния частиц, в частности задача о рассеянии электронов проводимости в бинарном металлическом сплаве на атомах примесей. Состояние электронов проводимости в квантовой механике описывается волновой функцией $\psi(\vec{r})$. Уравнения задачи формулируются для волновой функции $\psi(\vec{r})$ электрона в форме интегрального уравнения Шрёдингера. За нулевое приближение принимается волновая функция $\psi_{0}(\vec{r})$ электронов в совершенном кристалле одного из компонентов сплава в отсутствие атомов примесей других компонентов. Наличие атомов других компонентов сплава рассматривается как возмущение с известным потенциалом. Уравнение для волновой функции этой задачи имеет вид (оно называется также уравнением Корринги-Кона-Ростокера) [7,8]:

$$
\psi(\vec{r})=\psi_{0}(\vec{r})+\int_{V} d \vec{r}_{1} G\left(\vec{r}-\vec{r}_{1}\right)\left[\sum_{i=1}^{N} w_{i}\left(\vec{r}_{1}\right)\right] \psi\left(\vec{r}_{1}\right),
$$

где $w_{i}(\vec{r})$ - потенциал взаимодействия электрона с $i$-тым атомом примеси, быстро убывающая с расстоянием функция, суммирование производится по всем атомам примесей, а $G(\vec{r})$ - функция Грина уравнения Шрёдингера в отсутствие примесей.

В математическом отношении структуры основных уравнений статистической физики и механики гетерогенных сред во многом аналогичны. В обоих случаях осуществляется переход от дифференциальных уравнений в частных производных к интегральным уравнениям и решение последних методами теории возмущений (разложения по малому параметру, последовательных приближений). В настоящей работе предпринята попытка применить методы теории поля для описания состояния каждого отдельного кристаллита, а макродеформирование многокомпонентной поликристаллической среды рассмотреть как суммарный эффект коллективного поведения системы взаимодействующих кристаллитов.

1. Теоретико-полевой формализм в теории упругости поликристаллической среды. С точки зрения механики деформируемого твердого тела поликристаллический агрегат представляет собой многосвязную область объёмом $\Omega$ с внешней поверхностью $\Gamma$, состоящую из подобластей (кристаллитов) с объёмами $\omega_{\xi}$ и границами $\Gamma_{\xi}, \Omega=\sum_{\xi=1}^{N} \omega_{\xi}$, индекс $\xi$ нумерует кристаллиты, $N$ - полное число кристаллитов в теле, по границам $\Gamma_{\xi}$ обеспечивается прочное сцепление кристаллитов. В пределах каждого кристаллита материал однороден, упругие свойства кристаллита описываются постоянным тензором $C_{i j m n}^{0(\xi)}$ в кристаллографической системе координат (с осями, параллельными осям упругой симметрии кристаллита). Если номера $\xi$ относятся к кристаллитам одного физического типа, то соответствующие модули $C_{i j m n}^{0(\xi)}$ совпадают, 
иначе - различаются. В глобальной (лабораторной) системе координат тензор модулей упругости отдельного кристаллита запишется в виде

$$
C_{i j m n}^{(\xi)}(\vec{r})=\alpha_{i p}^{(\xi)}(\vec{r}) \alpha_{j q}^{(\xi)}(\vec{r}) \alpha_{m r}^{(\xi)}(\vec{r})_{n s}^{(\xi)} \alpha_{n s}^{(\xi)}(\vec{r}) C_{p q r s}^{0(\xi)},
$$

где $\alpha_{i p}^{(\xi)}(\vec{r})$ - направляющие косинусы осей лабораторной системы координат относительно кристаллографической системы координат $\xi$-того кристаллита.

Тензор модулей упругости поликристаллической среды имеет вид

$$
C_{i j m n}(\vec{r})=\sum_{\xi=1}^{N} \lambda_{\xi}(\vec{r}) C_{i j m n}^{(\xi)}(\vec{r})
$$

где $\lambda_{\xi}(\vec{r})$ - индикаторная функция $\xi$-того кристаллита:

$$
\lambda_{\xi}(\vec{r})= \begin{cases}1, & \text { если } \vec{r} \in \omega_{\xi} \quad \text { (т.е. принадлежит } \xi \text {-му кристаллиту); } \\ 0, & \text { в других случаях. }\end{cases}
$$

Тензор $C_{i j m n}(\vec{r})$ постоянен в каждой области $\omega_{\xi}$ и скачкообразно изменяется при переходе через границу $\Gamma_{\xi}$ вследствие смены типа кристаллита и изменения ориентации в пространстве кристаллической решётки кристаллита.

Краевая задача теории упругости гетерогенного тела состоит из уравнений равновесия, определяющих соотношений и граничных условий. С помощью введения функции Грина (тензора Кельвина-Сомильяны) эта дифференциальная форма краевой задачи приводится к эквивалентному интегральному уравнению для тензора деформаций:

$$
\varepsilon_{i j}(\vec{r})=\varepsilon_{i j}^{*}+\int_{V} d \vec{r}_{1} g_{i j k l}\left(\vec{r}-\vec{r}_{1}\right) C_{k l m n}^{\prime}\left(\vec{r}_{1}\right) \varepsilon_{m n}\left(\vec{r}_{1}\right),
$$

где $g_{i j k l}=\left(G_{i k, j l}+G_{j k}, i l\right) / 2$ - симметризованный тензор Грина, а $G_{i k, j l}-$ вторая производная тензора Грина (Кельвина-Сомильяны) краевой задачи теории упругости однородной среды с осреднённым тензором модулей упругости, $C_{k l m n}^{\prime}(\vec{r}) \equiv C_{k l m n}(\vec{r})-\left\langle C_{k l m n}\right\rangle-$ флуктуации тензора модулей упругости относительно осреднённого тензора

$$
\left\langle C_{i j k l}\right\rangle=\frac{1}{V} \int_{V} C_{i j k l}(\vec{r}) d \vec{r}=3\langle K\rangle V_{i j k l}+2\langle\mu\rangle D_{i j k l}
$$

$\left(V_{i j k l}\right.$ и $D_{i j k l}$ - шаровая и девиаторная части единичного симметричного тензора 4-го ранга), $\varepsilon_{i j}^{*}$ - макроскопическая однородная деформация тела. Тензор $g_{i j k l}$ имеет вид

$$
g_{i j k l}(\vec{r})=-\frac{1}{3\langle\mu\rangle}\left[(1-\chi) V_{i j k l}+\left(1-\frac{2}{5} \chi\right) D_{i j k l}\right] \delta(\vec{r})+\frac{F_{i j k l}(\theta, \varphi)}{r^{3}},
$$

где $\delta(\vec{r})$ - дельта-функция Дирака, $\chi=(3\langle K\rangle+\langle\mu\rangle) /(3\langle K\rangle+4\langle\mu\rangle), F_{i j k l}(\theta, \varphi)-$ функция только полярного $\theta$ и азимутального $\varphi$ углов радиус-вектора $\vec{r}$, явное выражение для которой приведено в [4]. 
Подставив (2) в (3), получим уравнение для деформаций неоднородной среды в виде

$$
\varepsilon_{i j}(\vec{r})=\varepsilon_{i j}^{*}+\int_{V} d \vec{r}_{1} g_{i j k l}\left(\vec{r}-\vec{r}_{1}\right)\left[\sum_{\xi=1}^{N} \lambda_{\xi}\left(\vec{r}_{1}\right)\left(C_{k l m n}^{(\xi)}\left(\vec{r}_{1}\right)-\left\langle C_{k l m n}\right\rangle\right)\right] \varepsilon_{m n}\left(\vec{r}_{1}\right) .
$$

Математически уравнение (5) в точности аналогично уравнению (1) для волновой функции электронов проводимости в сплаве.

В электронной теории твёрдых тел (в частности сплавов) очень хорошие результаты (по точности описания квантовых процессов) дает приближение сильной связи. Согласно этому приближению, области ненулевых значений волновых функций электронов сосредоточены в малых окрестностях узлов кристаллической решётки. Тогда в левой части уравнения (1) можно оставить значение волновой функции в каком-либо узле, а правая часть получится в виде суммы членов с волновыми функциями во всех остальных узлах. Каждый член этой суммы описывает взаимодействие (рассеяние) с полем соответствующего атома в узле. При сильном убывании взаимодействия с расстоянием между атомами основной вклад даёт член с тем же атомом, что и в левой части уравнения. Если пренебречь остальными членами, то получается так называемое одноузельное приближение в физике твёрдого тела, одно из простейших приближений. Следующие по величине вклады дают члены с соседними атомами - приближение ближайших соседей, и т.д.

Обратимся к поликристаллическому упругому телу. Аналогично представлению модулей упругости всего тела в виде суммы модулей кристаллитов (2) можно разложить тензоры деформаций и напряжений

$$
\varepsilon_{i j}(\vec{r})=\sum_{\xi=1}^{N} \lambda_{\xi}(\vec{r}) \varepsilon_{i j}^{(\xi)}(\vec{r}), \quad \sigma_{i j}(\vec{r})=\sum_{\xi=1}^{N} \lambda_{\xi}(\vec{r}) \sigma_{i j}^{(\xi)}(\vec{r})
$$

Учитывая, что объём тела равен сумме объёмов всех кристаллитов $\Omega=$ $=\sum_{\xi=1}^{N} \omega_{\xi}$, подставляя $(6)$ в $(5)$ и умножая обе части на $\lambda_{\xi}(\vec{r})$, получим

$$
\begin{aligned}
& \varepsilon_{i j}^{(\xi)}(\vec{r}) \lambda_{\xi}(\vec{r})=\varepsilon_{i j}^{*} \lambda_{\xi}(\vec{r})+ \\
& \quad+\lambda_{\xi}(\vec{r}) \int_{\omega_{\xi}} d \vec{r}_{1} g_{i j k l}\left(\vec{r}-\vec{r}_{1}\right)\left[\lambda_{\xi}\left(\vec{r}_{1}\right)\left(C_{k l m n}^{(\xi)}\left(\vec{r}_{1}\right)-\left\langle C_{k l m n}\right\rangle\right)\right] \varepsilon_{m n}^{(\xi)}\left(\vec{r}_{1}\right)+ \\
& \quad+\lambda_{\xi}(\vec{r}) \sum_{\eta \neq \xi} \int_{\omega_{\eta}} d \vec{r}_{1} g_{i j k l}\left(\vec{r}-\vec{r}_{1}\right)\left[\lambda_{\eta}\left(\vec{r}_{1}\right)\left(C_{k l m n}^{(\eta)}\left(\vec{r}_{1}\right)-\left\langle C_{k l m n}\right\rangle\right)\right] \varepsilon_{m n}^{(\eta)}\left(\vec{r}_{1}\right) .
\end{aligned}
$$

Для каждого $\varepsilon_{i j}^{(\xi)}(\vec{r})$ радиус-вектор $\vec{r}$ изменяется только в пределах $\xi$-того кристаллита. Отметим это обстоятельство дополнительным индексом $\xi$ у $\vec{r}$ : $\vec{r}_{\xi}$. Тогда уравнение $(7)$ перепишется (принимая во внимание, что $\lambda_{\xi}\left(\vec{r}_{\xi}\right)=1$ ) так:

$$
\varepsilon_{i j}^{(\xi)}\left(\vec{r}_{\xi}\right)=\varepsilon_{i j}^{*}+\int_{\omega_{\xi}} d \vec{r}_{\xi}^{\prime} g_{i j k l}\left(\vec{r}_{\xi}-\vec{r}_{\xi}^{\prime}\right)\left[\left(C_{k l m n}^{(\xi)}\left(\vec{r}_{\xi}^{\prime}\right)-\left\langle C_{k l m n}\right\rangle\right)\right] \varepsilon_{m n}^{(\xi)}\left(\vec{r}_{\xi}^{\prime}\right)+
$$




$$
+\sum_{\eta \neq \xi} \int_{\omega_{\eta}} d \vec{r}_{\eta}^{\prime} g_{i j k l}\left(\vec{r}_{\xi}-\vec{r}_{\eta}^{\prime}\right)\left[\left(C_{k l m n}^{(\eta)}\left(\vec{r}_{\eta}^{\prime}\right)-\left\langle C_{k l m n}\right\rangle\right)\right] \varepsilon_{m n}^{(\eta)}\left(\vec{r}_{\eta}^{\prime}\right)
$$

Уравнение (8) гласит, что деформация в точке $\vec{r}$ внутри $\xi$-того кристаллита определяется «взаимодействием» с деформациями в других точках данного кристаллита (второй член в правой части уравнения) и с деформациями в других кристаллитах (третий член в правой части). Это означает, что в отношении упругих свойств поликристаллический агрегат аналогичен системе многих взаимодействующих частиц, к которому можно приложить простые физические соображения о взаимодействии кристаллитов. Интегральное ядро в уравнении (8) согласно теоретико-полевой терминологии можно назвать пропагатором деформаций. С математической точки зрения (8) есть система $6 N$ интегральных уравнений для $6 N$ неизвестных функций $\varepsilon_{i j}^{(\xi)}\left(\vec{r}_{\xi}\right)$. Для решения подобных уравнений в статистической физике твёрдых тел разработано несколько хорошо зарекомендовавших себя методов.

2. Однокристаллитное приближение. Точное решение системы уравнений (8) является нереальной задачей. Для поликристаллов можно применить приём, аналогичный одноузельному приближению в теории рассеяния электронов на атомах примесей в кристаллической решётке металлического сплава, когда учитывается многократное рассеяние на одном узле и пренебрегается рассеянием на всех остальных. Для всех точек $\vec{r}$ внутри $\xi$-того кристаллита пренебрежём всеми членами под знаком суммы в (8), что соответствует пренебрежению взаимодействием с соседними и более далекими кристаллитами. Этому есть и формально математические предпосылки, т. к. функция $g_{i j k l}(\vec{r})$ быстро убывает с расстоянием пропорционально $1 / r^{3}$. Система (8) расщепляется на $N$ независимых подсистем из шести интегральных уравнений для компонентов тензора деформаций в каждом кристаллите

$$
\varepsilon_{i j}^{(\xi)}\left(\vec{r}_{\xi}\right)=\varepsilon_{i j}^{*}+\int_{\omega_{\xi}} d r_{\xi}^{\prime} g_{i j k l}\left(\vec{r}_{\xi}-\vec{r}_{\xi}^{\prime}\right)\left[C_{k l m n}^{(\xi)}\left(\vec{r}_{\xi}\right)-\left\langle C_{k l m n}\right\rangle\right] \varepsilon_{m n}^{(\xi)}\left(\vec{r}_{\xi}^{\prime}\right) .
$$

В пределах каждого кристаллита тензор $C_{k l m n}^{(\xi)}\left(\vec{r}_{\xi}\right)$ постоянен. При переходе к другому кристаллиту тензор скачкообразно изменяется вследствие поворота кристаллографических осей кристаллита и смены физического типа кристаллита - в случае многофазных поликристаллов.

Интегральные уравнения (9) трудно решать из-за наличия в функции Грина слагаемого $F_{i j k l}(\theta, \varphi) / r^{3}$. Примем дополнительное упрощающее предположение - пренебрежем неоднородностью деформаций внутри отдельного кристаллита, т. е. примем, что тензор $\varepsilon_{i j}^{(\xi)}\left(\vec{r}_{\xi}\right)$ постоянен в области $\vec{r}_{\xi} \subset \omega_{\xi}$. Тогда $\varepsilon_{i j}^{(\xi)}\left(\vec{r}_{\xi}\right)$ выносится из-под знака интеграла и система уравнений $(9)$ принимает вид

$$
\begin{array}{r}
\varepsilon_{i j}^{(\xi)}=\varepsilon_{i j}^{*}+\int_{\omega_{\xi}} d r_{\xi}^{\prime}\left\{-\frac{1}{3\langle\mu\rangle}\left[(1-\chi) V_{i j k l}+\left(1-\frac{2}{5} \chi\right) D_{i j k l}\right] \delta\left(\vec{r}_{\xi}-\vec{r}_{\xi}^{\prime}\right)+\right. \\
\left.+\frac{F_{i j k l}(\theta, \varphi)}{\left|\vec{r}_{\xi}-\vec{r}_{\xi}^{\prime}\right|^{3}}\right\} C_{k l m n}^{\prime(\xi)} \varepsilon_{m n}^{(\xi)} .
\end{array}
$$


В интеграле, содержащем $F_{i j k l}(\theta, \varphi) /\left|\vec{r}_{\xi}-\vec{r}_{\xi}^{\prime}\right|^{3}$, мысленно выделим малую шаровую область с центром в точке $\vec{r}_{\xi}$ и целиком находящуюся внутри $\xi$-того кристаллита. Легко показать, что если в этой области сначала произвести интегрирование по углам, то соответствующий интеграл обращается в нуль, устраняя сингулярность $1 / r^{3}$ в точке $\vec{r}_{\xi}$. Оставшийся интеграл по объёму кристаллита $\omega_{\xi}$ вне выделенной шаровой области можно приближённо положить равным нулю вследствие быстрого убывания функции $F_{i j k l}(\theta, \varphi) /\left|\vec{r}_{\xi}-\vec{r}_{\xi}^{\prime}\right|^{3}$ с расстоянием от точки $\vec{r}_{\xi}$ и знакопеременности $F_{i j k l}(\theta, \varphi)$. Это созвучно известному сингулярному приближению в теории упругости неоднородных сред при вычислении эффективного модуля, заключающемуся в пренебрежении формальной составляющей функции Грина (4) после операции осреднения, но приводит, как показано ниже, к отличающимся результатам.

Оставшийся интеграл в уравнении (10) тривиально вычисляется и для каждого кристаллита получается система шести линейных уравнений для $\varepsilon_{i j}^{(\xi)}$ с постоянными коэффициентами:

$$
\begin{aligned}
\varepsilon_{i j}^{(\zeta)}=\varepsilon_{i j}^{*}+\left[\frac{1}{3}(4 \chi-1) V_{i j k l}+\right. & \frac{2}{3}(1 \\
& \left.-\frac{2}{5} \chi\right) D_{i j k l}+ \\
& \left.+\frac{\chi}{5\langle\mu\rangle} V_{i j m n} C_{m n k l}^{(\xi)}-\frac{1-\frac{2}{5} \chi}{3\langle\mu\rangle} C_{i j k l}^{(\xi)}\right] \varepsilon_{k l}^{(\zeta)} .
\end{aligned}
$$

Отличие систем (11) для разных кристаллитов сводится к отличию в значениях направляющих косинусов осей кристаллографических систем координат кристаллитов и (для многофазных кристаллов) к смене тензора модулей упругости $C_{\text {pqrs }}^{0(\xi)}$.

Выражение в квадратных скобках (11) есть некоторый тензор, который в данной модели определяет состояние $\xi$-того кристаллита, постоянен в области $\xi$-того кристаллита, а совокупность всех этих тензоров задаёт структуру поликристалла. Всего имеется $N$ таких тензоров, для которых введём отдельное обозначение:

$A_{i j k l}^{(\zeta)}=\left[\frac{1}{3}(4 \chi-1) V_{i j k l}+\frac{2}{3}\left(1-\frac{2}{5} \chi\right) D_{i j k l}+\frac{\chi}{5\langle\mu\rangle} V_{i j m n} C_{m n k l}^{(\xi)}-\frac{\left(1-\frac{2}{5} \chi\right)}{3\langle\mu\rangle} C_{i j k l}^{(\xi)}\right]$ Тогда уравнение (11) перепишется так:

$$
\left[I_{i j k l}-A_{i j k l}^{(\xi)}\right] \varepsilon_{k l}^{(\zeta)}=\varepsilon_{i j}^{*}
$$

Если тензор в квадратных скобках (12) обратим, то решение системы уравнений (12) можно написать в общем виде:

$$
\varepsilon_{i j}^{(\zeta)}=\left[I_{i j k l}-A_{i j k l}^{(\xi)}\right]^{-1} \varepsilon_{k l}^{*}
$$

Решение системы (13) нельзя получить в аналитическом виде даже для кристаллитов простейших (с высокой симметрией) сингоний. Однако линейная система всегда может быть решена численно. 
От полученных таким способом массивов значений деформаций можно перейти к массивам значений напряжений в кристаллитах по формулам

$$
\sigma_{i j}^{(\xi)}=C_{i j k l}^{(\xi)}\left(\vec{r}_{\xi}\right) \varepsilon_{k l}^{(\zeta)}, \quad \xi=1,2, \ldots, N
$$

Совокупность решений для полей $\varepsilon_{i j}^{(\zeta)}$ и $\sigma_{i j}^{(\xi)}$ полностью определяет состояние отдельных кристаллитов при заданном макроскопическом состоянии поликристалла, задаваемом макродеформацией $\varepsilon_{k l}^{*}$. Макронапряжения, соответствующие заданному полю $\varepsilon_{k l}^{*}$, вычисляются по очевидным формулам:

$$
\left\langle\sigma_{i j}\right\rangle=\frac{1}{\Omega} \int_{\Omega} \sigma_{i j}^{(\xi)}(\vec{r}) d \vec{r}=\frac{1}{\Omega} \sum_{\xi=1}^{N} \omega_{\xi} \sigma_{i j}^{(\xi)}
$$

Совокупность уравнений (13)-(15) полностью описывает упругое деформирование поликристалла как агрегата взаимодействующих кристаллитов. При увеличении макродеформаций $\varepsilon_{k l}^{*}$ в некоторых кристаллитах (в зависимости от их типа) могут начаться процессы хрупкого повреждения, пластического или вязкоупругого течения и другие. Это может быть учтено сменой определяющих соотношений (14) для таких кристаллитов. При этом макронапряжённое состояние поликристалла по-прежнему определяется соотношением (15), но связь макронапряжений с макродеформацией становится нелинейной. В [9] продемонстрировано появление макроскопической псевдопластичности при учёте хрупкого разрушения зерен поликристалла вследствие флуктуаций напряжений с помощью более громоздкого метода. В [10] совокупность систем уравнений (11) была применена для вычисления функций распределения случайных деформаций и напряжений в упругом однофазном поликристалле.

Подставив (13) в (14), получим выражение для напряжений в кристаллитах в форме

$$
\sigma_{i j}^{(\xi)}=C_{i j k l}^{(\xi)}\left(\vec{r}_{\xi}\right)\left[I_{k l m n}-A_{k l m n}^{(\xi)}\right]^{-1} \varepsilon_{m n}^{*}, \quad \xi=1,2, \ldots, N
$$

которая содержит характеристики только $\xi$-того кристаллита. Если сравнить его с напряжениями в приближении Фойгта

$$
\sigma_{i j}^{(\xi)}=C_{i j m n}^{(\xi)}\left(\vec{r}_{\xi}\right) \varepsilon_{m n}^{*}, \quad \xi=1,2, \ldots, N
$$

которое соответствует полному пренебрежению взаимодействием кристаллитов, то видно, что учёт взаимодействия кристаллитов сводится к перенормировке тензоров модулей упругости всех кристаллитов. Это является аналогом ситуации в физике конденсированного состояния, когда система многих взаимодействующих частиц с массами $m_{\xi}$ может быть представлена в виде системы невзаимодействующих квазичастиц, но с перенормированными массами $\tilde{m}_{\xi}$, определяемыми по формулам вида

$$
\tilde{m}_{\xi}=m_{\xi} /\left(1+\alpha_{\xi}\right),
$$


где $\alpha_{\xi}$ - перенормировочная поправка, аналогом которой здесь выступает тензор $A_{k l m n}^{(\xi)}$.

В настоящей работе ограничимся приложением изложенного формализма к классической задаче вычисления эффективных модулей неоднородных материалов.

3. Приложение к теории эффективного модуля. Әффективный (макроскопический) тензор модулей упругости $C_{i j k l}^{*}$ связывает осреднённые тензоры деформаций $\left\langle\varepsilon_{k l}\right\rangle$ и напряжений $\left\langle\sigma_{i j}\right\rangle$ в поликристалле:

$$
\left\langle\sigma_{i j}\right\rangle=C_{i j k l}^{*}\left\langle\varepsilon_{k l}\right\rangle, \quad\left\langle\varepsilon_{k l}\right\rangle=\varepsilon_{k l}^{*} .
$$

Вычисление эффективных модулей сводится к вычислению средних напряжений в теле по тем или иным моделям.

Для макроскопически изотропного многофазного поликристалла, состоящего из $n$ физически различных типов кристаллитов (ориентации осей симметрии всех кристаллитов равновероятны, а форма и размеры случайны), в случае выполнения эргодической гипотезы и возможности использования концепции представительного элемента объёма среды в рамках предлагаемого подхода средние напряжения по всему поликристаллу определяются выражением

$$
\begin{aligned}
\left\langle\sigma_{i j}\right\rangle= & \frac{1}{\Omega} \int_{\Omega} \sigma_{i j}^{(\xi)}(\vec{r}) d \vec{r}=\frac{1}{\Omega} \sum_{\xi=1}^{N} \int_{\omega_{\xi}} \sigma_{i j}^{(\xi)}\left(\vec{r}_{\xi}\right) d \vec{r}_{\xi}= \\
= & \frac{1}{\Omega}\left[\sum_{\eta=1}^{N_{1}} \omega_{\eta}^{(1)} C_{i j k l}^{(1)}\left(\vec{r}_{\eta}\right) \varepsilon_{k l}^{(1)}\left(\vec{r}_{\eta}\right)+\sum_{\eta=1}^{N_{2}} \omega_{\eta}^{(2)} C_{i j k l}^{(2)}\left(\vec{r}_{\eta}\right) \varepsilon_{k l}^{(2)}\left(\vec{r}_{\eta}\right)+\cdots+\right. \\
& \left.+\sum_{\eta=1}^{N_{n}} \omega_{\eta}^{(n)} C_{i j k l}^{(n)}\left(\vec{r}_{\eta}\right) \varepsilon_{k l}^{(n)}\left(\vec{r}_{\eta}\right)\right]
\end{aligned}
$$

где $N$ - полное число кристаллитов в поликристаллическом теле, верхний индекс у тензоров $C_{i j k l}$ и $\varepsilon_{k l}$ в последнем равенстве обозначает физический тип кристаллита; $N_{1}, N_{2}, \ldots, N_{n}$ - числа кристаллитов типов $1,2, \ldots n ; N=$ $=N_{1}+N_{2}+\ldots+N_{n}$.

Если число кристаллитов каждого типа достаточно велико, то все суммы в правой части (17) можно представить в виде

$$
\begin{aligned}
\frac{1}{\Omega} \sum_{\eta=1}^{N_{1}} \omega_{\eta}^{(1)} C_{i j k l}^{(1)}\left(\vec{r}_{\eta}\right) \varepsilon_{k l}^{(1)}\left(\vec{r}_{\eta}\right) & =\frac{\Omega^{(1)}}{\Omega} \sum_{\eta=1}^{N_{1}} \frac{\omega_{\eta}^{(1)}}{\Omega^{(1)}} C_{i j k l}^{(1)}\left(\vec{r}_{\eta}\right) \varepsilon_{k l}^{(1)}\left(\vec{r}_{\eta}\right)= \\
& =\frac{\Omega^{(1)}}{\Omega} \sum_{\eta=1}^{N_{1}} \pi_{\eta}^{(1)} C_{i j k l}^{(1)}\left(\vec{r}_{\eta}\right) \varepsilon_{k l}^{(1)}\left(\vec{r}_{\eta}\right)=v_{1}\left\langle C_{i j k l}^{(1)}\left(\vec{r}_{\eta}\right) \varepsilon_{k l}^{(1)}\left(\vec{r}_{\eta}\right)\right\rangle_{1},
\end{aligned}
$$

где $\pi_{\eta}^{(1)} \equiv \omega_{\eta}^{(1)} / \Omega^{(1)}$ означает вероятность того, что кристаллит типа 1 имеет некоторую заданную ориентацию кристаллографических осей; $v_{1}=\Omega_{1} / \Omega$ - 
объёмная доля кристаллитов типа $1 ;\langle\cdots\rangle_{1}$ означает условное среднее только по областям объёмов кристаллитов первого типа, и аналогично для остальных типов кристаллитов. При введении условных средних выражение для средних напряжений по всему объёму поликристалла (безусловных средних) перепишется так:

$$
\left\langle\sigma_{i j}\right\rangle=\sum_{\eta=1}^{n} v_{\eta}\left\langle C_{i j k l}^{(\eta)}\left(\vec{r}_{\eta}\right)\left[I_{k l m n}-A_{k l m n}^{(\eta)}\right]^{-1}\right\rangle_{\eta} \varepsilon_{m n}^{*} .
$$

Из (16) следует формула для эффективного тензора модулей упругости многофазного поликристалла в рассмотренном приближении

$$
C_{i j m n}^{*}=\sum_{\eta=1}^{n} v_{\eta}\left\langle C_{i j k l}^{(\eta)}\left(\vec{r}_{\eta}\right)\left[I_{k l m n}-A_{k l m n}^{(\eta)}\right]^{-1}\right\rangle_{\eta}
$$

Для кристаллитов произвольной сингонии соотношения (18) можно использовать только для численной реализации. Для сравнения предлагаемого подхода с традиционными рассмотрим модельную систему - статистическую смесь $n$ изотропных компонентов с объёмными долями $v_{1}, v_{2}, \ldots, v_{n}$ и модулями упругости

$$
C_{i j k l}^{(1)}=3 K_{1} \cdot V_{i j k l}+2 \mu_{1} \cdot D_{i j k l}, \quad \ldots, \quad C_{i j k l}^{(n)}=3 K_{n} \cdot V_{i j k l}+2 \mu_{n} \cdot D_{i j k l} .
$$

Осреднённый тензор модулей упругости составляет величину

$$
\left\langle C_{i j k l}\right\rangle=3\langle K\rangle \cdot V_{i j k l}+2\langle\mu\rangle \cdot D_{i j k l},
$$

где $\langle K\rangle=v_{1} K_{1}+v_{2} K_{2}+\ldots+v_{n} K_{n},\langle\mu\rangle=v_{1} \mu_{1}+v_{2} \mu_{2}+\ldots+v_{n} \mu_{n}$. В этом случае перенормировочный тензор упрощается, в (18) легко производится обращение тензоров, а для тензора эффективных модулей (изотропного в данном случае) получается выражение

$$
C_{i j k l}^{*}=3 K^{*} V_{i j k l}+2 \mu^{*} D_{i j k l}, \quad K^{*}=\sum_{\eta=1}^{n} v_{\eta} \tilde{K}_{\eta}, \quad \mu^{*}=\sum_{\eta=1}^{n} v_{\eta} \tilde{\mu}_{\eta},
$$

где

$$
\tilde{K}_{\eta}=\frac{K_{\eta}}{1+\frac{(1-\chi)\left(K_{\eta}-\langle K\rangle\right)}{\langle\mu\rangle}}, \quad \tilde{\mu}_{\eta}=\frac{\mu_{\eta}}{1+\frac{2(1-2 / 5 \cdot \chi)\left(\mu_{\eta}-\langle\mu\rangle\right)}{3\langle\mu\rangle}}
$$

- перенормированные модули компонентов смеси.

Выражения (19), (20) отражают упомянутую выше перенормировку модулей в системе взаимодействующих кристаллитов в терминах эффективных модулей. Полному пренебрежению взаимодействием кристаллитов соответствует приближение Фойгта, в котором эффективные модули равны средним модулям:

$$
K_{\text {Фойгт }}^{*}=\langle K\rangle=v_{1} K_{1}+v_{2} K_{2}+\ldots+v_{n} K_{n} .
$$

Учёт взаимодействия кристаллитов формально сводится к замене в (21) упругих модулей изолированных компонентов $K_{\eta}$ и $\mu_{\eta}$ на перенормированные $\tilde{K}_{\eta}$ и $\tilde{\mu}_{\eta}$. 
Формулы (19), (20) отличаются от известных формул корреляционного и сингулярного приближений в теории упругости неоднородных сред [4].

Заключение. В работе отмечена глубокая математическая аналогия уравнений механики неоднородных сред и квантовой теории конденсированного состояния в теоретико-полевой формулировке. Это позволяет применить идеи и приближённые методы решения уравнений квантовой теории к решению уравнений механики. В настоящей работе для вычисления деформаций и напряжений в поликристаллических зёрнах материала использован приближённый метод, основанный на простейшем приближении квантовой теории рассеяния, применяемом в физике твёрдого тела для описания состояний электронов проводимости в металлических сплавах, - одноузельном приближении. В применении к механике поликристаллов это соответствует учёту взаимодействия деформаций в пределах одного кристаллита и пренебрежению всеми остальными. В этом приближении деформированное состояние поликристаллического тела представляется в виде суперпозиции состояний невзаимодействующих кристаллитов, но с перенормированными модулями. В данной работе возможности предлагаемого подхода проиллюстрированы на примере классической задачи вычисления эффективных модулей упругости неоднородной среды. Однако основная цель метода - прослеживание эволюции состояний индивидуальных кристаллитов и их влияния на макродеформирование поликристаллического материала. Но это не является целью данной работы и представляет собой отдельное научное исследование.

Работа выполнена при поддержке РФФИ (проект №13-01-96052).

\section{БИБЛИОГРАФИЧЕСКИЙ СПИСОК}

1. W. Voight, Lehrbuch der kristallphysik (mit ausschluss der kristalloptik). Leipzig - Berlin: B. G. Teubner, 1928. 978 pp.

2. G. I. Taylor, "Plastic Strain in Metals"// J. Inst. Met., 1938. Vol.62. Pp. 307-324 (Reprinted in the Scientific Papers of G. I. Taylor. 1. Cambridge University Press, Cambridge, U.K., 1958).

3. И. М. Лифиич, Л. Н. Розенцвейг, "К теории упругих свойств поликристаллов" // Ж. эксn. теор. физ., 1946. T. 16, №11. C. 967-980. [I. M. Lifshits, L. N. Rozentsveig, "Theory of elastic properties of polycrystals" // Zh. Eksp. Teor. Fiz., 1946. Vol. 16, no.11. Pp. 967980].

4. Т. Д. Шермергор, Теория упругости микронеоднородных сред. М.: Наука, 1977. 400 с. [T. D. Shermergor, Theory of elasticity of micro-inhomogeneous media. Moscow: Nauka, 1977. 400 pp.]

5. Л. Д. Ландау, Е. М. Лифиии, Теоретическая физика. Т. 9: Статистическая физика. Часть 2. Теория конденсированного состояния. М.: Наука, 1978. 448 с. [E. M. Lifshits, Theoretical physics. Vol. IX: Statistical physics. Part 2. The theory of a condensed state. Moscow: Nauka, 1978. 448 pp.]

6. А. А. Абрикосов, Л. П. Горъков, И. Е. Дзялошинский, Методы квантовой теории поля в статистической физике. М.: Физматлит, 1962. 443 с. [A. A. Abrikosov, L. P. Gor'kov, I. E. Dzjaloshinskiy, Methods of quantum field theory in statistical physics. Moscow: Fizmatlit, 1962. 443 pp.]

7. Neil W. Ashcroft, N. David Mermin, Solid State Physics. Fort Worth: Saunders College, 1976. 826 рр.; русск. пер.: Н. Ашкрофт, Н. Мермин, Физика твердого тела (в 2-х томах). Т. 1. М.: Мир, 1979. 399 с.; Т. 2. М.: Мир, 1979. 422 с.

8. Л. И. Ястребов, А. А. Кацнельсон, Основы одноэлектронной теории твердых тел. М.: 
Наука, 1981. 320 с.; англ. яз.: L. I. Yastrebov, A. A. Katsnelson, Foundations of OneElectron Theory of Solids / AIP Translation Series. Berlin: Springer, 1987. 335 pp.

9. V. Shavshukov, A. Tashkinov, Y. Strzhemechny, D. Hui, "Modelling of pseudoplastic deformation of carbon/carbon composites with a pyrocarbon matrix" // Modelling Simul. Mater. Sci. Eng., 2008. Vol. 16, no. 5, 055001. 18 pp.

10. В. Е. Шавшуков, "Распределение полей напряжений в поликристаллических материалах" // Физ. мезомех., 2012. Т. 15, №6. С. 85-91. [V. E. Shavshukov, "Stress field distribution in polycrystalline materials" // Fiz. Mezomekh., 2012. Vol.15, no.6. Pp. 85$91]$.

Поступила в редакцию 11/IX/2013;

в окончательном варианте - 15/X/2013.

MSC: 74N15; 74E15, 74M25

\section{FIELD-THEORETIC APPROACH FOR CHARACTERIZATION THE DEFORMATION OF MULTICOMPONENT POLYCRYSTALLINE MATERIALS}

\section{A. A. Tashkinov, V. E. Shavshukov}

Perm National Research Polytechnic University, 29, Komsomolskiy Prospekt, Perm, 614990, Russia.

E-mails: tash@pstu.ru; shavshukov@pstu.ru

Most of inorganic structural materials (metallic alloys, ceramics, minerals etc.) are polycrystalline aggregates, consisted of macroscopically large quantity of single-crystal grains (crystallites). The mechanical behavior of the specimen of polycrystalline material is governed by the physical and mechanical processes in the grains and interaction of the grains. Thus the deformation of polycrystalline material is a cooperative phenomenon typical for condensed matter physics and mechanics of heterogeneous materials. The passing of these processes depends on many parameters, including stress states of individual grains and its evolution during macrodeformation. In this paper we note a mathematical analogy between the equations of the mechanics of heterogeneous polycrystalline materials and the equations of quantum theory of particles scattering. This analogy allows to apply the methods of quantum field theory to solution of the equations of solid mechanics for heterogeneous media. We consider the application of Corringa-Kohn-Rostoker method, used in quantum theory for calculating wave function of electrons in metallic alloys, to elasticity of polycrystals. This approach allows, for instance, to calculate probability distribution density function for stresses in grains under arbitrary macrodeformation of polycrystal. Application of the method to classical problem of homogenization gives new formulae for the effective moduli of disordered polycrystalline medium.

Keywords: quantum field theory, polycrystals, stress, strain, tensor of effective elastic moduli.

Original article submitted 11/IX/2013;

revision submitted $15 / \mathrm{X} / 2013$.

Anatoliy A. Tashkinov (Dr. Phys. \& Math. Sci.), Rector; Head od Dept., Dept. of Mechanics for Composite Materials and Structures. Vyacheslav E. Shavshukov (Ph. D. Phys. \& Math.), Associate Professor, Dept. of Mechanics for Composite Materials and Structures. 